\title{
Symmetric Networks Foster to Evolve Desirable Turn-taking Rules in Dispersion Games
}

\author{
Akira Namatame, Hiroshi Sato, Member, IEEE
}

\begin{abstract}
Using a game-theoretic model combined with the evolutionary model, we investigate the conditions under which the desirable interaction rules will evolve and sustain in various social interaction settings. The direction of the research to come is to understand how the interaction structure, the network topology, determines the path of evolutionary dynamics. For the emergence of desirable outcomes at the macroscopic level, the patterns of social interaction are critical. We find that the efficient and fair outcome emerges relatively quickly in symmetric networks where each agent plays the game with the same number of players. In symmetric networks, agents appear to easily recognize the possibility of a coordinated turn-taking behaviour or alternating reciprocity as a means to generate an efficient and fair outcome.
\end{abstract}

\section{INTRODUCTION}

$\mathrm{E}$ VOLUTIONARY game theory offers one approach to the study of emergent properties in systems of interacting agents. Darwinian dynamics based on mutation and selection form the core of models for evolution in nature. Evolution through natural selection is understood to imply improvement and progress. If a population of agents is adapting each other, the result is a co-evolutionary process. The problem to contend with in co-evolution based on the Darwinian paradigm, however, is the possibility of an escalating arms race with no end. Competing agents might continually adapt to each other in more and more specialized ways, never stabilizing at a desirable outcome. Of particular interests is the question how social interactions can be structured so that agents are free to choose their own actions while avoiding outcomes that none would have chosen. Social norms are often understood as solutions to coordination problems that arise in a population of individuals large enough that most members never directly interact with one another, thus precluding the possibility of effective collective action.

Previous research on evolution of social norms has mainly focused on the Prisoner's Dilemma (PD) game. The conflict between individual rationality and collective rationality problem has been largely solved by the theories of reciprocal altruism and indirect reciprocity, and computer simulations have shown that reciprocal strategies such as Tit for Tat (TFT) tend to evolve, resulting in widespread joint cooperation. The evolutionary model is to explain how TFT could have evolved as social norms, given that natural selection operates at the level of the individuals.

Kandori et al., (1993) studied the evolution of play in a

Akira Namatame and Hiroshi Sato are with the Computer Science Department, National Defense Academy, Yokosuka, JAPAN( e-mail: (nama, hsato)@nda.ac.jp) population of players continually interacting with coordination games. Players are repeatedly and randomly matched against opponents that are known as random matching model. Players revise their strategic choices only at discrete random moments, each player independent of the others. But the dynamics of social interaction limit what kind of coordination can occur. For the pair-wise coordination problems studied, the only emergent state is risk-dominant coordination. However, there is no reason to believe that risk-dominant selection is universal. Hanaki et al., (2005), for instance, have shown that in at least some models where matching is endogenous, payoff-dominant selection can obtain.

A major shortcoming of the previous influential research is its focus on games in which cooperation in Prisoner's Dilemma (PD) game or coordination in coordination games involves the agents acting similarly. There are games in which favorable payoffs are possible only if one player acts one way while the other acts the opposite way. For instance, to cooperate successfully, the agents have to alternate or take turns, out of phase with each other. A typical example is the battle of sexes game. If this type of interaction is repeated, then the agents benefit, in terms of natural selection, by coordinated alternation by taking turns in choosing one of the two strategies and there is evidence to show that this type of turn-taking occurs quite commonly in nature. Give and take or alternation is a behavioural rule that is intuitive and simple, but even so it is beyond the scope of most traditional evolutionary models.

Browning et al., (2004) investigate through agent-based simulation how this type of coordinated, alternating cooperation can evolve without any communication between players. Using a genetic algorithm, they showed that coordinated turn-taking can evolves in games with asymmetric Nash equilibria if the players benefit from it. However, precisely how coordination evolves without communication is not fully explained.

Hanaki (2006) used adaptive models to understand the dynamics that lead to efficient and fair outcomes in the repeated battle of sexes game. He develops a model that not only uses reinforcement learning but also the evolutionary learning that operates through evolutionary selection. He found that the efficient and fair outcome emerges relatively quickly through turn taking. However, his model requires a long run pre-experimental phase before it is ready to take turn. Turn taking in the battle of the sexes game is just one of many game theoretic phenomena, and it raises an important general point for further studies.

In this paper, we study the nature, properties and phenomena of coordinated alternating cooperation in a range of games with asymmetric equilibria and to attempt 
to find a mechanism to explain how it evolves. We especially study how the interaction structure, the network topology determines to foster desirable evolutionary outcomes. For the emergence of desirable outcomes at the aggregate level, the patterns of social interactions are critical. We investigate how the efficient and fair outcome emerges relatively quickly in symmetric situations where each agent plays the game with the same number of players. In symmetric networks, agents appear to easily recognize the possibility of a coordinated turn-taking behaviour with coordinated alternating reciprocity as a means to generate an efficient and fair outcome.

\section{DISPERSION GAMES}

We consider a population of $\mathrm{N}$ agents, each faces a binary choice problem between two behavioral types: Cooperate (C) or Defect (D). For any agent the payoff to a choice of $\mathrm{C}$ or $\mathrm{D}$ depends on how many other agents also choose $\mathrm{C}$ or $\mathrm{D}$. Here we consider social interactions in which agents are identically situated in the sense that every agent's outcome, whichever way she makes her choice, depends on the number of agents who choose on way or the other. The payoff to each agent is given as an explicit function of the actions of all agents, and therefore she has an incentive to pay attention to the collective decision. The payoffs to each agent choosing from Cooperate (C) or Defect (D) are given:

$$
\begin{aligned}
& U(C)=(a-b) p+b, \\
& U(D)=(c-d) p+d
\end{aligned}
$$

where $p(0 \leq p \leq 1)$ is the proportion of the agents who choose $\mathrm{D}$ (defect). The binary decision itself can be analyzed by decomposing into the underlying $2 \mathrm{x} 2$ games with the payoff matrix in Table I with the condition, $c>$ a, $\mathrm{b}>\mathrm{d}$.

A more studied class of games is the coordination games in which agents gain high payoffs when they choose the same action. A complementary class that has received relatively little attention is the games in which agents gain payoffs only when they are disperse by choosing distinct actions. These games are sometimes called the dispersion games. The two payoff functions can be equated at the intersection and such $p$ is obtained as

$$
p=(b-d) /(b+c-a-d) \equiv \theta \text {. }
$$

The dispersion game has the unique equilibrium in which both strategies are used at the ratios $\theta$ and $1-\theta$. Under this mixed population, the payoffs of all agents are equal. If the game is repeated, there will be a tendency towards a stable equilibrium with $\mathrm{N}$ agents choosing $\mathrm{C}$ and the rest (1- ) N agents choosing $\mathrm{D}$.

In the context of the two-person symmetric game with the payoff matrix in Table I, a pair of mixed strategy $(\boldsymbol{p}, \boldsymbol{p})$ constitutes a Nash equilibrium. A Nash equilibrium of the two-person game is defined in terms of how the payoff is affected when she switch to the other strategy when the other agent sticks to the current mixed strategy $\boldsymbol{p}=(p$, $1-p)$.However, such a mixed equilibrium situation is not to be socially efficient. Any agent who chooses $\mathrm{C}$ or $\mathrm{D}$ gains if
TABLE I

THE PAYOFF MATRIX OF A SOCIAL GAME

\begin{tabular}{c|c|c}
\hline $\begin{array}{c}\text { All others' choices } \\
\text { Own choice }\end{array}$ & $\begin{array}{c}\text { Cooperate(C) } \\
(p)\end{array}$ & $\begin{array}{c}\text { Defect (D) } \\
(1-p)\end{array}$ \\
\hline Cooperate (C) & $a$ & $b$ \\
\hline Defect (D) & $c$ & $d$ \\
\hline
\end{tabular}

some choosing D will shift and choose C. The collective maximum occurs to the left of the intersection. Since the slope of the payoff function $C$ is shaper than that of the payoff function of $\mathrm{D}$, if fewer agents than the ratio at the intersection choose $\mathrm{C}$, agents who choose $\mathrm{C}$ will gain more than the loss of the agents who choose $\mathrm{D}$. If the collective maximum does not occur at the intersection, there is a payoff difference between a choice of $C$ and a choice of $D$. For instance, if the collective maximum occurs to the left of the intersection, agents who choose $\mathrm{D}$ gain less than those who choose $C$. This is a big difference from a Nash equilibrium at the intersection in which all agents receive the same payoff.

Collective efficiency (Pareto efficiency) is achieved at the strategy distribution where the average payoff per agent is maximized. We denote the average payoff per agent by $E(p)$ when the strategy distribution is $p=(p, 1-p)$. Since the proportion of agents to choose $C$ is $p$ and that of agents to choose $D$ is $1-p$, the average payoff per agent is

$$
\begin{aligned}
E(p) & =p U\left(e_{1}, p\right)+(1-p) U\left(e_{2}, p\right) \\
& =(a+d-b-c) p^{2}+(b+c-2 d) p+d
\end{aligned}
$$

Collective efficiency is achieved under the strategy population $\boldsymbol{p}=\left(p^{*}, 1-p^{*}\right)$ where $p^{*}$ is given as

$$
p^{*}=(b+c-a-d) / 2(b+c-a-d) \text {. }
$$

There is often more than one Pareto outcome, not every Pareto outcome will be regarded as desirable. In general there are many Pareto efficient allocations, some of which are very bad from the point of view of equity, and there is no connection between Pareto efficiency and equity. In particular, a Pareto efficient outcome may be very inequitable.

\section{EVOLUTION OF BEHAVIOURAL RULES}

The literature on learning in the game theory is mainly concerned with the understanding of learning procedures that if adopted by interacting agents will converge in the end to the Nash equilibrium of the underlying game. The main concern is to show that adaptive dynamics lead to a rational behaviour, as prescribed by a Nash equilibrium strategy. We call a dynamical system uncoupled if an agent's learning model does not depend on the payoff functions of the other agents. Hart (2003) proved that there are no uncoupled dynamics that are guaranteed to converge to Nash equilibrium. Therefore, a coupling between agents, that is, the adjustment of an agent's strategy depends on the payoff functions of the other agent, is a basic condition for convergence to Nash equilibrium.

The learning algorithms themselves are not required to satisfy any rationality requirement. Instead, they converge to a rational behaviour if it is adopted by all agents (Young 2005). In addition, Nash equilibrium cannot make precise 
TABLE II

LEARNABLE BEHAVIOURAL RULE (\# REPRESENTS 0 OR 1)

\begin{tabular}{c|c|c|c}
\hline $\begin{array}{c}\text { Strategy site in } \\
\text { Fig. } 1\end{array}$ & \multicolumn{2}{|c|}{ Previous strategies } & \multirow{2}{*}{ Next strategy } \\
\cline { 2 - 3 } & Own & Opp & \\
\hline 4 & 0 & 0 & $\#$ \\
\hline 5 & 0 & 1 & $\#$ \\
\hline 6 & 1 & 0 & $\#$ \\
\hline 7 & 1 & 1 & $\#$ \\
\hline
\end{tabular}

TABLE III

ALL POSSIBLE BEHAVIOURAL RULE S WITH HISTORY 1

\begin{tabular}{ll|ll}
\hline Type 1: 0000 & (ALL-C) & Type 9: 0001 & \\
Type 2: 1000 & & Type10: 1001 & \\
Type 3: 0100 & & Type11: 0101 & (TFT) \\
Type 4: 1100 & & Type12: 1101 & \\
Type 5: 0010 & & Type13: 0011 & \\
Type 6: 1010 & & Type14: 1011 & \\
Type 7: 0110 & (PAVLOV) & Type15: 0111 & (FRIEDMAN) \\
Type 8: 1110 & & Type16: 1111 & (ALL-D) \\
\hline
\end{tabular}

predictions about the outcome of repeated games. Nor can it tell us much about the dynamics by which a collective of agents moves from an inefficient equilibrium to a better outcome. These limitations, along with concerns about the cognitive demands of forward-looking rationality, have motivated many researchers to explore alternatives backward-looking learning models. Most of these efforts have been invested in evolutionary dynamics. The research agenda of evolutionary dynamics is to explore non-equilibrium explanations of equilibrium in repeated games to view equilibrium as the long-run outcome of a dynamic evolutionary process.

In this paper, we will take a different approach from the previous evolutionary dynamics by focusing on collective evolution. This approach differs from the common use of the genetic algorithm, in which the goal is to optimize a fixed fitness function. In the genetic algorithm, the focus is also on the best final result. In collective evolution, we are interested in a better way of coupling among agents, which leads to desirable joint actions.

The first question we must address is what individuals know and what it is that they are learning about. In repeated games, agents repeatedly play an underlying game, each time observing their payoff and other agents' strategies. In the classic work on learning in game theory, the agents select their strategy in the next iteration of the game based on the result of the previous play using some updating rule. In the repeated model, agents engage in a series of games with different rules at each stage. In fact, the nature of each game depends on the results of the pervious game, and this means the strategy choice depends on agents' joint action in the previous rounds of games.

An important aspect of iterated games is the introduction of a behavioural rule by which an agent can decide her strategy (Lindgren 1997). We will shift attention to coupled dynamics among agents with behavioural rules. We make a distinction between adaptive dynamics and coupled dynamics. In an adaptive dynamics, other mechanisms are allowed as well, e.g., modifications of strategies based on

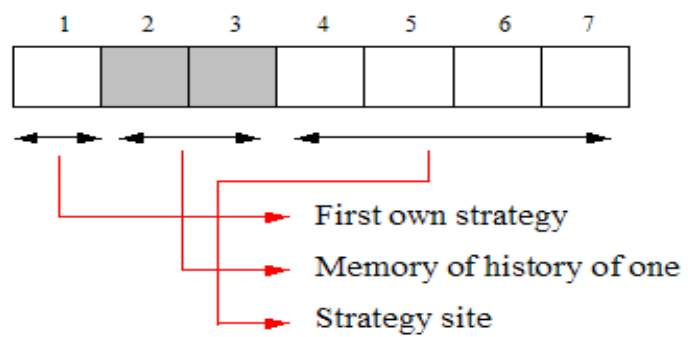

Fig. 1 A behavioural rule based on the history $1(\mathrm{~h}=1)$

the strategy distribution of the population. But, such adaptive dynamics do not necessarily improve the outcome to which the individual belongs in the long run. Evolutionary dynamics refer to the systems based on the basic mechanisms of biological evolution, that allows, inheritance, mutation, and selection. However, evolutionary dynamics based on natural selection also converge to an inefficient outcome. Coupled evolutionary dynamics differ, in this sense, from evolutionary dynamics, in which a fixed goal is used in the fitness function and where there is no coupling among agents.

Let assume that each agent remembers the past $h$ outcomes (history). A behavioural rule must specify, for each history, what strategy the agent should choose. We represent $C$ by 0 and $D$ by 1 as shown in Table II. In Table III, we show all possible behavioural rules with $\mathrm{h}=1$. There are four possible outcomes for each move between two agents:(0, 0), $(0,1),(1,0)$, and $(1,1)$. A quick calculation shows that the number of possible behavioural rules with the outcome of only the previous round is 16 . With the increase of the memories of the past rounds, there are a huge number of behavioural rules. The hope is that agents would find a better behavioural rule out of the overwhelming number of possible rules after a reasonable number of repeated games.

Each behavioural rule specifies the strategy choice based on the outcome of the previous round. Agent strategies are restricted to those employing only the previous move with the other agent to determine the next choice. Each agent has an evolvable rule as shown in Fig. 1. Since no memory exists at the start, an extra one bit are needed to specify a hypothetical history.

\section{REPEATED DISPERSION GAMES ON NETWORKS}

It is important to consider with whom an agent interacts and how each agent decides her action depending on others' actions. In order to describe interaction at the individual level, we may have two fundamental models, global interaction (or mean-field model) and local interaction. The introduction of spatial dimensions, so that individuals only interact with those in their neighbourhood, may affect the dynamics of the system in various ways. The possibility of space-temporal structures may allow for global stability where the mean-free model would be unstable. The presence of these various forms of space-temporal phenomena may, therefore, also alter the evolutionary path compared with the mean-field case and we may see other strategies evolve.

The introduction of spatial interactions leads to the 
d
iI

d

d

C

n

C.

iI

S1

n

O

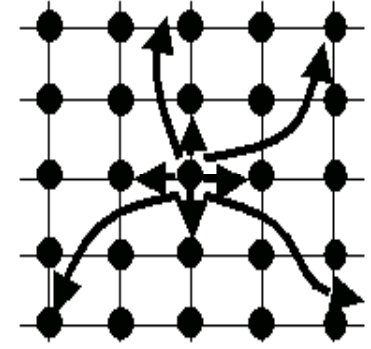

(b)

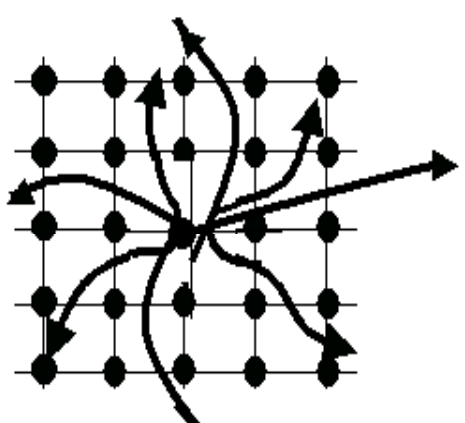

(c) ir Fig. 2 Repeated games on a grid. (a) Games on local networks (symmetric rl nearest neighbors.(b) Games on a small-world network (asymmetric mode e nearest neighbors and four other agents randomly chosen from the populat sı (asymmetric model). Each agent interacts with her eight randomly chosen hıgnest scorıng inaıviaual or the nearest neignoours. I nese offspring play the same strategy as their ancestors, unless a mutation occurs, which happens at a small mutation rate. If a mutation occurs, the offspring's strategy is not its parent's strategy but a new strategy chosen randomly.

The main effect of the spatial structure in the repeated $\mathrm{PD}$, for instance, is that cooperative strategies can build clusters in which the benefits of mutual cooperation can outweigh losses against defectors. Thus, clusters of cooperators can invade groups of defectors that prevail in non-spatial populations. The selection pressure of such an arrangement is clearly lower, since individuals are only assessed on a local level, not in a global fashion. This allows for individuals, which may have been eliminated if assessed against all players, to survive in a niche eventually be fit individuals or contribute genetic material to fit individuals as the environment changes. More importantly, from the viewpoint of a truly evolutionary system (one in which the individuals are evolving, as well as the population), the use of this spatial arrangement is thought to increase the genetic diversity by preserving apparently less fit strategies in niches.

Recent studies on the structure of social, technological, and biological networks have shown that they share salient features that situated them far from being completely regular or random. Social interactions are rarely well described by random or regular networks. Therefore, we also need to study the influence of the topological aspects of networks by exploring the different network topology. The topology of social networks is much better described by what has been called a small-world network (Watts 2003), as shown in Fig. 2(b).

In a regular lattice model, agents interact with the nearest neighbours. In the version of a small-world network, a fraction of the neighbours is replaced by breaking interactions. An equal number of new agents are selected from outside of the current neighbours. These new agents for interaction are selected randomly from the rest of the population. Kirley (2000) studied an evolutionary version of the prisoner's dilemma game, played by agents placed in a small-world network. Agents are able to change their strategy, imitating that of the most successful neighbour. They found that collective behaviours corresponding to the small-world network enhances defection where cooperation is 1

Another imp dynamic entitie of agents that studied the evc agent plays : neighbours. $\mathrm{T}$. adapting to th simple setting (Fig. 3. The average payoff per agent over generation which there is $r$ interaction lin interaction evi that governs th the resulting small-world $\mathrm{n} \epsilon$ selection is int

Various stu network struct of iterated cor partners with networks that

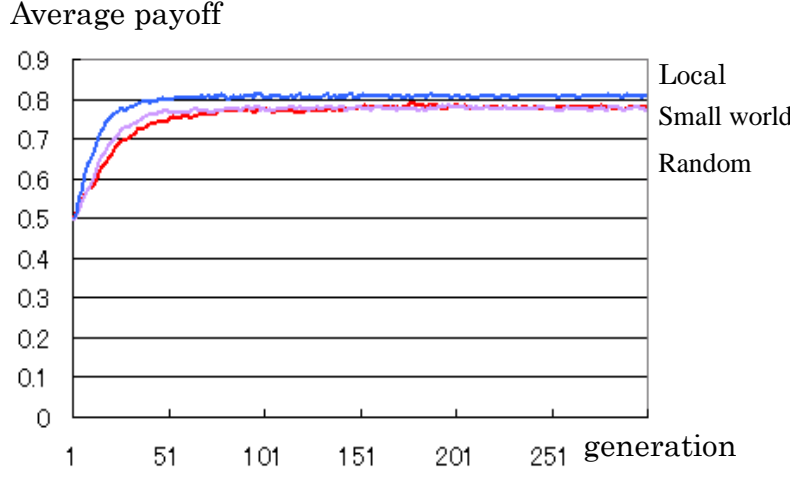
underlying coordination game. Ellison (1993) analyzed the role of local interactions for the spread of particular strategies in coordination games, showing how play converges to risk-dominant equilibrium if agents are located on a circle and interact with their two nearest neighbours. Similarly, Blume (1993) and Kosfeld (2002) proved the convergence to the risk-dominant equilibrium in a population of agents located on a two-dimensional lattice. 
Goyal et al., (2005) studied the formation of networks among agents who are bilaterally involved in coordination games. In addition to specifying which pairs of agents in the population play the game, the network structure also determines how strategic information diffuses among the agents and how coordination among the agents is found. They showed that once agents are allowed to choose their partners, the situation is very different. They introduce a number of locations where agents can meet and play the coordination game with each other. Thus, at any time, agents choose both a location and a strategy in the game. Under these conditions they showed that risk dominance looses its selection force and that the population is most likely to coordinate on the Pareto efficient equilibrium. Since agents can freely choose their interaction partners, they are able to find partners that choose the Pareto efficient equilibrium strategy, and at the same time they can avoid agents that choose the risk-dominant inefficient strategy.

Browning et al., (2004) investigated how this type of coordinated, alternating cooperation can evolve without any communication between agents who play the dispersion game. Using a genetic algorithm incorporating mutation and crossing-over, they showed that coordinated turn-taking can evolves in battle of sexes and chicken games with asymmetric Nash equilibria. The procedure followed Wu et al., (1997). For each outcome of the game, each agent receives one of four payoffs, and she remembers three past outcomes. Since there are 43 different three-move histories, each string of 64 binary digits suffices to specify a choice for every three-move history.

The offspring rules that played in each subsequent generation were formed from the most successful rule of the previous generation, using a genetic algorithm. The algorithm implemented the following five steps: (1) The payoff values were assigned according the underlying game. (2) An initial population was for each of the 20 randomly chosen rules. (3) In each generation, each of the 20 rules was paired with each of the others for the fixed number of repetitions with every other rule in the population (global interaction). (4) At the end of each generation, after each rule had played with each of the others, each rule's mean payoff was computed, and it was as signed a mating probability proportional to its fitness score. (5) For each offspring strategy, two rules were randomly selected as parents, selection being proportional to mating probability scores.

We will compare and identify the effect of the manner of interaction by considering the lattice network, small-world network, and random network as shown in Fig. 2. In the local model, each agent interact his/her nearest eights agents (Fig. 2(a)). In the small-world network (Fig. 2(b)), the locally networked agents will be compared to a half-mixed population in which a half of the population is again modeled by a lattice, but in this case each agent interacts with four partners that are nearest neighbours and four partners that are randomly chosen from the population. In the random matching model (Fig. 2(c)), each agent interact with eight other agents chosen randomly. We compare and identify the effect of the manner of interaction

by considering evolved behavioural rules. In small-world network, for instance, The locally networked agents will be compared to a half-mixed population in which a half of the population is again modelled by a lattice, but in this case each agent interacts with four partners that are nearest neighbours and four partners that are randomly chosen from the population.

\section{THE EFFECTS OF THE NETWORK TOPOLOGY}

In this section we discuss the impact of different network structures on evolution of social norms in the context of iterated games. If agents can choose the partners with which to interact, then they will form networks that lead to efficient Nash equilibrium play in the underlying games.

\section{A. Symmetric Dispersion Game}

First of all, we consider the case in which all agents repeatedly play the symmetric dispersion game with the payoff matrix in Table IV. This game has two equilibria with the pairs of the pure strategies $(C, D),(D, C)$ and one equilibrium of the mixed strategy.

The average payoff per agent at each generation is shown Fig. 3. Fig. 3 shows the same experiment, this time using different network frameworks. There is little difference in the graphs using local and small-world networks and the graph obtained using the non-spatial random environment.

The advantage of agent-based modelling is that we can investigate behavioural rules learned by all agents that lead to a desirable collective outcome at the macro level. In Table V, we show the behavioural rules learned by 2,500 agents. In the beginning, they are endowed with randomly chosen behavioural rules. However, these different rules were updated through collective evolution. The 2,500 rules were finally aggregated into a few types, as shown in Table $\mathrm{V}$. The numbers in the right-hand column represent the number of agents who share the same rule. Those aggregated rules have the commonality. We show the strategy choices between two agents with the rules in Table $\mathrm{V}$ as the state transition process as shown in Fig. 4. If agents

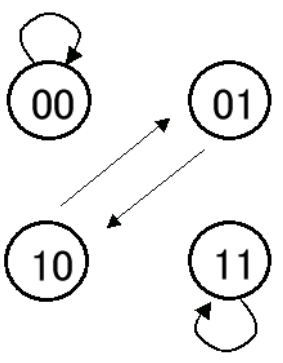

Case 1

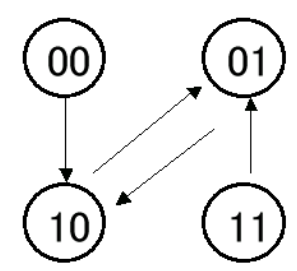

Case 2

Fig. 6. State transition of evolved behavioural rules in Table 5. Case1: Two agents with the same behavioural rule, Case2: Two agents with different behavioural rules

choose $\mathrm{C}$ and their opponent chooses $\mathrm{D}$ at the previous time period, then they choose C. If agents choose D and their opponent chooses $\mathrm{C}$ at the previous time period, then they choose D. These rules represent the following action: if they gain then they repeat the same winning strategy, and this is the same principle of reinforcement learning. 
TABLE VI

PAYOFF MATRIX OF THE ASYMMETRIC DISPERSION GAME

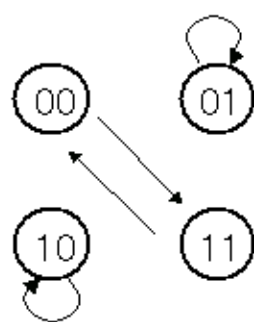

Case 1
Case 2

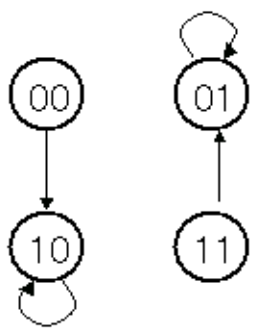

rules in Table 5: Case1: Two agents with the same behavioural rule, Case2: Two agents with different behavioural rules

TABLE V.

BEHAVIOURAL RULES LEARNED BY 2,500 AGENTS IN THE SYMMETRIC DISPERSION GAME: LATTICE OR SMALL-WORLD NETWORK

\begin{tabular}{|c|c||c|c|c|c|c|}
\hline \multirow{2}{*}{$\begin{array}{c}\text { Rule } \\
\text { type }\end{array}$} & \multirow{2}{*}{$\begin{array}{c}\text { Initial } \\
\text { strateg } \\
\text { y }\end{array}$} & 4 & 5 & 6 & 7 & $\begin{array}{c}\text { Number } \\
\text { of agents }\end{array}$ \\
\hline \hline 5 & 0 or 1 & 0 & 0 & 1 & 0 & 699 \\
\hline 6 & 0 or 1 & 1 & 0 & 1 & 0 & 805 \\
\hline 13 & 0 or 1 & 0 & 0 & 1 & 1 & 383 \\
\hline 14 & 0 or 1 & 1 & 0 & 1 & 1 & 613 \\
\hline
\end{tabular}

Next, we investigate the case in which the underlying game is the asymmetric dispersion game with the payoff matrix in Table VI. Fig. 5 shows the average payoff per agent and it was gradually increased to 1.6. Fig. 5 shows the same experiment, this time using different network frameworks. There are a couple of important differences in this graph and the graph obtained using the non-spatial random environment. Under the random network model, the average payoff per agent is about 0.6.

In Table VII, we show the behavioural rules learned by 2,500 agents. In the beginning, they are endowed with randomly chosen behavioural rules. These different rules were updated through collective evolution and they were finally aggregated into a few types in Table VII. The numbers in the right-hand column represent the number of agents who share the same rule. Those aggregated rules have the commonality. We show the strategy choices between two agents with the rules in Table VII as the state transition process in Fig. 6.

There is a significant difference depending on two agents learn to have the same rule or different rules. If two agents with the same rule interact, they absorb in to $(0,0)$ or $(1,1)$ as shown in Fig. 6 (Case 1). On the other hand, if they have different rules, the turn-taking behaviour is emerged as shown in Fig. 6 (Case 2). If one agent chooses $C$ and their opponent chooses $\mathrm{D}$ at the previous time period, then they choose C. If agents choose D and their opponent chooses C at the previous time period, then they choose $\mathrm{D}$. These rules represent the following action: if they gain then they repeat the same winning strategy, and this is the same principle of reinforcement learning.

Most agents succeeded in learning the behavioural rules that have the following property. If the agent gains the

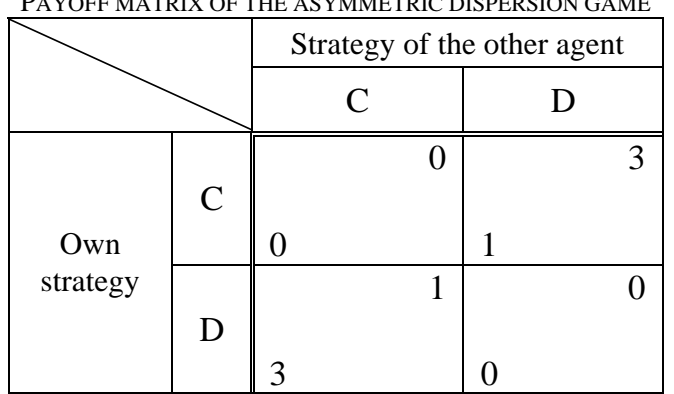

Average payoff

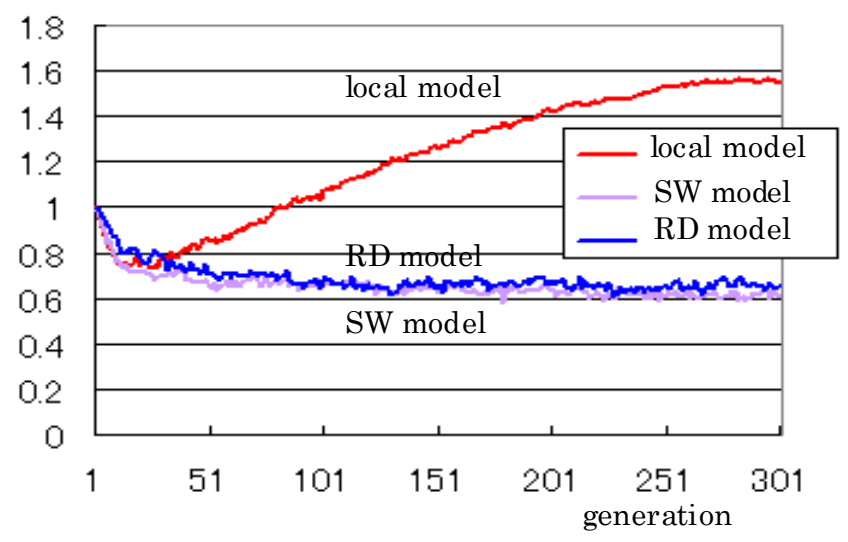

Fig. 5 The average payoff per agent over generations

TABLE VII

BEHAVIOURAL RULES LEARNED BY 2,500 AGENTS: THE ASYMMETRIC DISPERSION GAME AND VARIANT PD GAME UNDER LOCAL MODEL

\begin{tabular}{|c|c||c|c|c|c|c|}
\hline \multirow{2}{*}{$\begin{array}{c}\text { Rule } \\
\text { type }\end{array}$} & \multirow{2}{*}{\begin{tabular}{c} 
Initial \\
strateg \\
\cline { 3 - 6 }
\end{tabular}} & 4 & 5 & 6 & 7 & $\begin{array}{c}\text { Number } \\
\text { of agents }\end{array}$ \\
\hline \hline 3 & 1 & 0 & 1 & 0 & 0 & 335 \\
\hline 4 & 1 & 1 & 1 & 0 & 0 & 430 \\
\hline 11 & 0 or 1 & 0 & 1 & 0 & 1 & 919 \\
\hline 12 & 0 & 1 & 1 & 0 & 1 & 816
\end{tabular}

payoff (success), then they change their strategies. We can characterize that this behavioural rule is based on the principle of so-called give-and-take When agents face symmetric dispersion games with the payoff matrix in Table $\mathrm{V}$, there is no difference in the payoff under outcome $(C, D)$ or $(D, C)$. Therefore, they learn the behavioural rules to continue the same strategy if they gain.

On the other hand, when they face asymmetric dispersion games with the payoff matrix in Table VI, the payoffs to both agents at the two pure Nash equilibria (C, D) and (D, C) become asymmetric. Therefore, they learn to realize efficient and equitable outcomes by visiting the two pure Nash equilibria alternatively.

They showed that about $85 \%$ of the plays in the population are characterized by coordinated turn taking. They study the nature, properties and phenomena of coordinated alternating cooperation in a range of dispersion games with asymmetric equilibria. By alternating coordination the agents benefit from it, however, how 
TABLE VIII.

BEHAVIOURAL RULES LEARNED BY 2,500 AGENTS: THE ASYMMETRIC DISPERSION GAME UNDER THE SMALL WORLD NETWORK AND RANDOM MODELS.

\begin{tabular}{|c|c||c|c|c|c|c|}
\hline \multirow{2}{*}{$\begin{array}{c}\text { Rule } \\
\text { type }\end{array}$} & \multicolumn{1}{|c||}{\begin{tabular}{c} 
Initial \\
strateg \\
\cline { 3 - 6 }
\end{tabular}} & 4 & 5 & 6 & 7 & $\begin{array}{c}\text { Number } \\
\text { of agents }\end{array}$ \\
\hline \hline 5 & 1 & 0 & 0 & 1 & 0 & 719 \\
\hline 6 & 1 & 1 & 0 & 1 & 0 & 380 \\
\hline 13 & 0 & 0 & 0 & 1 & 1 & 842 \\
\hline 14 & 0 & 1 & 0 & 1 & 1 & 559 \\
\hline
\end{tabular}

agents evolves alternating coordination without communication is not fully explained.

In Table VIII, we show the behavioural rules learned by 2,500 agents who repeatedly play the asymmetric dispersion games in Table VIII under the small-world and random networks. In the beginning, they are endowed with randomly chosen behavioural rules. However, these different rules were updated through collective evolution. 2,500 rules were finally aggregated into four types, as shown in Table VIII. The numbers in the right-hand column represent the number of agents who share the same rule. Those aggregated rules are different from those rules under local model and small-world network as shown in Table VII.

Consensus problems and synchronization have a long history in computer science, control theory, and physics. (Olfati-Sabe, Fax\&Murray 2007) (Moft 2005). In networks of agents, consensus means to reach an agreement regarding a certain quantity of interest that depends on the state of all agents. A consensus algorithm is an interaction rule that specifies the information exchange between an agent and all of its neighbors on the network. The consensus and synchronization process also relate to emergence of coherent patterns.

In this case, a kind of networks where the underling network topology is balanced (symmetric networks) is a necessary condition for emergence of coordination. As a result, we now have a better understanding of emergence of desirable aggregate process. How we act is often influenced by neighbors, where the network topology determines important form of interactions. We discuss that topological properties of network should be derived from the dynamics by which the networks are created. Having a large number of interactions is not enough by itself to guarantee emergence. The diffusion process may need to reach a certain critical mass as well as the connectivity should be symmetric before desirable behavioural rules emerge.

\section{CONCLUSION}

The direction of the research to come was to understand how the structure of social networks determines emergence and the diffusion dynamics of social norm. For the emergence, the patterns of social interactions are critical. For the diffusion at the social level, the network topology is so important. As a result, we now have a better understanding of desirable emergence and diffusion process. How we act is often influenced by acquaintances or neighbors, where the social networks determine important form of social interaction. We discuss that topological properties of network should be derived from the diffusion dynamics by which better behavioural rules are created and diffused. Having a large number of interactions is not enough by itself to guarantee emergence and diffusion. The diffusion process may need to reach a certain critical mass, in some cases, a combined threshold of diversity, and connectivity before desirable emergent properties appears.

The other direction of the research was to understand how a collection of agents arrives at specific desirable collective outcomes. The first priority for a desirable collective outcome is stability, which is crudely modelled using the idea of equilibrium of an underlying game. The next priority is efficiency, which is also defined as following Pareto optimality and is equivalent to the requirement that nobody can be made better off without someone else being made worse off. The third priority is equity. We aim to discover the fundamental behavioural rules that generate the desirable macroscopic structures of interest. This type of self-organization is referred as the emergence of desired orders from the bottom up.

Many sphere of social interactions are governed by norms such as reciprocity and equity. Social norms and conventions are the glue that holds a society of self-interested agents together. When social outcomes appear to be coordinated, the cause is most often not a remarkable concerted collective action, but individual adherence to well-understood and shared guides to normative behaviour. Although social norms can potentially serve useful constructs to understand human behaviour, there is little theory of norm development. Although social norms have been conceptualized in various ways by a variety of researchers, definition in a recent review article is as follows: Social norms are rules and standards that are understood by members of a group, and that guide and/or constrain social behaviour without the force of laws. These norms emerge out of interaction with others; they may or may not be stated explicitly, and any sanctions for deviating from them come from social networks, not the legal system. Put differently, norms are shared rules that emerge and are sustained through people's autonomous interaction without formal regulating authorities or forces such as laws. This definition highlights emergence and sustainability of social norms as core issues for any theory of norms.

In an evolutionary explanation of norm development, there is no need to assume a rational calculation to identify the effective rule. Instead, the analysis of what is chosen at any specific time is based upon an implementation of the idea that effective rules are more likely to be retained than ineffective ones. Furthermore each agent mimics the most successful neighbour as guidance of improving her behavioural rule. Their success depends in large part on how well they learn from their neighbours. If an agent gains more payoff than her neighbour, there is a chance her behavioural rule will be imitated by others. The more successful agents are more likely to survive and reproduce 
effective behavioural rules. However, agents also observe each other, and those agents with poor performance tend to imitate the rules of those they see doing better. This mechanism of collective evolution tends to evolve to both efficient and equitable outcomes. Furthermore, the asymmetry in payoffs from interaction induces agents to learn the behavioural rule, so-called turn-taking norm to break the asymmetry in social interactions.

\section{REFERENCES}

[1] Axelrod, R. The Complexity of Cooperation, Princeton University Press, 1997.

[2] Blume, L. E. "The Statistical Mechanics of Strategic Interaction.” Games and Economic Behavior Vol.4, 1993, p.387-424.

[3] Blume J., Durlauf,S., "Equilibrium Concepts for Social Interaction Models.”

[4] International Game Theory Review, Vol.5, No.3, 2003, p.193-210.

[5] Browning, L., Colman, M., "Evolution of coordinated alternating reciprocity in repeated dyadic games". Journal of Theoretical Biology Vol.229, 2004, p. 549-557

[6] Durkheim, E. The Rules of Sociological Method, The Free Press, 1982.

[7] Ellison, G.,'Learning local interaction, and coordination”, Econometrica, Vol.61, 1993, p.1047-1071.

[8] Goyal, S., Vega-Redondo, F.,’Learning, network formation and coordination”, Games and Economic Behavior Vol.50, 2005.

[9] Hanaki, N. , Erev,S.,I., Peterhansl, T., "Learning Strategies", Journal of Economic Behavior and Organization, Vol. 56, 2005.p.523-542.

[10] Hanaki, N.,'Individual and Social Learning”, Computational Economics, 2006

[11] Hart, S., Mas-Colell, A.,’Uncoupled dynamics do not lead to Nash equilibrium”, American Economic Review, Vol.93, 2003, p. 1830-1836.

[12] Kandori, M., Mailath, G.,and Rob,B.,. "Learning, Mutation and Long Run Equilibrium in Games.” Econometrica Vol.61, 1993, p.29-56.

[13] Kandori, M. "Social Norms and Community Enforcement.” The Review of Economic Studies, Vol.59, No1. 1992, p.63-80.

[14] Kirley, M.,”Compex networks and evolutionary games”. Proc. of the 7th Asia-Pacific Conf. On Complex Networks, 2004.

[15] Kosfeld, M.,’Stochastic strategy adjustment in coordination games”, Economic Theory, Vol. 20, 2002. p. 321-339.

[16] Lindgren, K.,"Evolutionary dynamics in game-theoretic models”, The Economy As An Evolving Systems II, Addison-Wesley, 1997.

[17] Motter, A, Zhou, C, and Kutth, J.(2005). Network synchronization, Diffusion, and the Paradox of Heterogeneity, Physical Review E. 71, 016116-1-9

[18] Namatame, A, Adaptation and Evolution in Collective Systems, World Scientific, 2006.

[19] Olfati-Saber R., Fax J.A., R.M. Murray. (2007). Consensus and Cooperation in Networked Multi-Agent Systems. Proceedings of the IEEE, Vol. 95, Jan. pp. 401-420

[20] Schelling, T. Micromotives and Macrobehavior, Norton,1978.

[21] Skyrms, B., Evolution of the Social Contract, Cambridge University Press, 1996.

[22] Tesfatsion, L., Ashlock, H., Smucker,.. "Preferential partner selection in an evolutionary study of prisoner's dilemma", BioSystems, Vol.37,1996, pp. 9-25,

[23] Young, H. P. “The Evolution of Conventions.” Econometrica Vol. 61, No.1,1993, p.57-84.

[24] Young, H. P., Individual Strategy and Social Structures, Princeton University Press, 1998

[25] Young, H. P. Strategic Learning and Its Limits, Oxford Univ. Press, 2005

[26] Zimmermann.,M.., and Eguíluz,V., "Cooperation, social networks, and the emergence of leadership in a prisoner's dilemma with adaptive local interactions, Physical Review, E, Vol.72, 2005, p.056118-1-15

[27] Watts, D. Small Worlds, Princeton University Press,1999.

[28] $\mathrm{Wu}$, J. and Axelrod, R. How to cope with noise in the iterated prisoner's dilemma game, Journal of Conflict Resolution, 39, 1997, p. 183-189. 